\title{
EXOGENOUS APPLICATIONS OF POTASSIUM DIHYDROGEN ORTHOPHOSPHATE AND SOWING DATES ENHANCE FRUIT YIELD AND ESSENTIAL OIL OF CORIANDRUM SATIVUM L.
}

\author{
E.Y. Abdul-Hafeez \\ Department of Floriculture, Faculty of Agriculture, Assiut University, Egypt
}

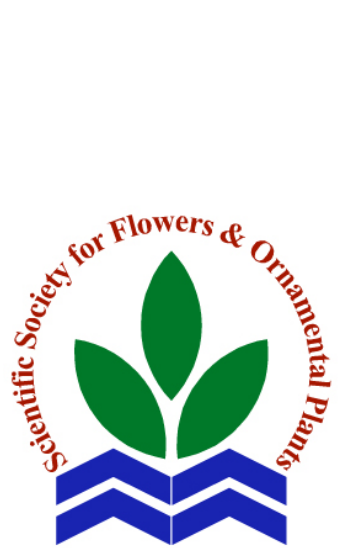

Scientific J. Flowers \& Ornamental Plants, 8(1):181-194 (2021).

Received:

$15 / 2 / 2021$

Accepted:

$25 / 3 / 2021$

Corresponding author: E.Y. Abdul-Hafeez eyhafeez@aun.edu.eg

ABSTRACT: A field experiment was conducted during two growing seasons to study the effect of foliar application of potassium dihydrogen orthophosphate $\left(\mathrm{KH}_{2} \mathrm{PO}_{4}\right)$ at different concentrations; 0 , $1000,2000,3000,4000 \mathrm{ppm}$, and sowing date $\left(15^{\text {th }}\right.$ October, $1^{\text {st }}$ November and $15^{\text {th }}$ November) on the growth, fruit yield, and essential oil productivity of coriander (Coriandrum sativum L.). Among the three sowing dates tested, plants sown on $1^{\text {st }}$ November recorded the best results regarding all characteristics during both seasons, except for plant height during the second season only and stem diameter during both seasons which were significantly increased for the plants sown on $15^{\text {th }}$ October. Plants grown on $15^{\text {th }}$ October significantly surpassed those grown on $15^{\text {th }}$ November in most of the studied parameters. Foliar application of $\mathrm{KH}_{2} \mathrm{PO}_{4}$ fertilizer exhibited a significant effect on plant height, branch number/plant, stem diameter, umbel number/plant, fruit dry weight per plant and per fed, harvest index, essential oil percentage and essential oil yield per plant and per fed. The highest concentration of $\mathrm{KH}_{2} \mathrm{PO}_{4}$ (4000 ppm) showed the best results of all studied parameters comparing to the control and the other concentrations in both seasons. The best results were noticed in the plants grown on $1^{\text {st }}$ November and treated with potassium at 4000 ppm in all parameters except for plant height which achieved the best combination when plants were sown on $15^{\text {th }}$ October and treated with potassium at $4000 \mathrm{ppm}$.

Key words: Coriander, Coriandrum sativum, potassium, essential oil, sowing date.

\section{INTRODUCTION}

Annual erect coriander plant (Coriandrum sativum L.) is cultivated and produced worldwide for culinary, aromatic and medicinal uses. It is also commonly referred to as coriander when grown for its herbs, and is used in many foods. The essential oil extracted from the fruits of coriander (common as seeds) has many uses (Diederichsen, 1996). Ground coriander seeds are used as a spice, for example in the preparation of curry. The essential oil is one of the main flavor compounds in gin. Additionally, coriander essential oil is used to flavor bread, sauces, soups, canned goods and desserts. It has shown antimicrobial characteristics on the growth of some fungi and bacteria such as Escherichia coli, Yersinia enterocolitian, Staphylococcus aureus and Rhodotorula sp. as they completely banned under in vitro conditions (Elgayyar et al., 2001). The essential oil of coriander is most commonly extracted from the fruits by either hydro or steam distillation. It was found that the content of essential oil in coriander seeds ranges between 0.125 and $1.90 \%$ (Jeliazkova et al., 1997; Lenardis et al., 2000; Ayanoglue et al., 2002; Gil et al., 2002), and main ingredient of the essential oil is linalool, it ranges from 40 to $82.9 \%$ of the oil (Machado et al., 1993; Diederichsen, 1996 and Pino et 


\section{E.Y. Abdul-Hafeez}

al., 1996). The other main components of seed oil are pinene, terpinene, camphor, geranyl acetate, geraniol, borneol, terpine-4ol, terpineol, citronellol and nerol, and limonene (Pino et al., 1996 and Gil et al., 2002).

Potassium plays an important role in various functions of plants: enzyme activation, photosynthesis, osmotic potential, protein synthesis, and as anti-ion to organic bio-polymers and inorganic ions (Britto and Kronzucker, 2008). With potassium deficiency, photosynthesis and nitrogen uptake were decreased (Peuke et al., 2002). Cao et al. (1993) reported that absence of potassium and ammonium inhibited root development in Arabidopsis and this influence could be counteracted by potassium possibly via activation of ammonium assimilatory enzymes (Hagin et al., 1990). Managing root of vegetables is somewhat more difficult than leafy vegetables because of the nutrient requirements of a plant change during the different phases of plant growth. From seed germination to root development, the plant needs are fairly constant. At the start of rooting, it needs more potassium. At this stage, potassium can be added to the agricultural soil or as a foliar application, which is authenticated by some researchers as a very effective method to nourish the plant during the phase of intensive growth (Chauduni and De, 1975 and Giskin et al., 1984). Leafy vegetables will be ready for foliar nutrition when the leaf expansion has reached an acceptable leaf area. Potassium dihydrogen orthophosphate $\left(\mathrm{KH}_{2} \mathrm{PO}_{4}\right)$, synonym potassium dihydrogen phosphate or monopotassium phosphate, is widely used as a foliar nutrient for plant growth. Many investigations showed that potassium is a strong mobile element that did not show a significant difference between soil and foliar supplies (Fan and Moshe, 2002; Awad et al., 2014 and Pande et al., 2014).

Changes in essential oil yield and composition have been reported to be influenced by cultivars and sowing dates
(Rangappa et al., 1997) and environmental conditions (Gil et al., 2002). The sowing date was found to affect plant biomass but not seed yield (Gil et al., 1999). A delayed date of planting accelerates subsequent development stages and shortens the entire plant vegetative growth period (Carrubba et al., 2006), thus reducing yields (Luayza et al., 1996; Carrubba et al., 2006 and Zheljazkov et al., 2008). Decreased yield of coriander plants as a result of delayed sowing is due to poor bud growth and lower yield components (Carrubba et al., 2006), that depend on plant's response to sunlight and the length of the day (Diederichsen, 1996 and Weiss, 2002). Thus, regardless of the grown species, the sowing date is considered to be a cost-free element in any vegetable production technology.

The purpose of this study was to determine the effect of potassium dihydrogen orthophosphate and different sowing dates as well as their interaction on growth, fruit yield and essential oil productivity of coriander (Coriandrum sativum L.) plants.

\section{MATERIALS AND METHODS}

This study was based on the results of an experiment on coriander designed at the Department of Floriculture, Assiut University, Egypt. Field trials were carried out during 2018/2019 and 2019/2020 seasons, on a field at the Floriculture Experimental Farm (N- 27.252 ; E-31.09 $)$. Maximum and minimum temperatures, as well as the relative humidity of the research location were obtained from the Meteorological Station at the Exper. Farm, Fac. of Agric., Assiut Univ. (Table, 1). The experiment was set up on a clayey soil. The soil physical and chemical characteristics of the experimental field were analyzed before the application in compliance with the methods cited by Jackson (1973) and Black et al. (1982), as shown in Table (2).

The aim of this investigation was to study the influence of potassium dihydrogen orthophosphate $\left(\mathrm{KH}_{2} \mathrm{PO}_{4}\right)$ (El-Nasr Co. for 
Table 1. Monthly average of metrological data of the experimental farm during 2018, 2019 and 2020 years.

\begin{tabular}{lcccccccccccc}
\hline & \multicolumn{4}{c}{$\mathbf{2 0 1 8}$} & \multicolumn{4}{c}{$\mathbf{2 0 1 9}$} & \multicolumn{4}{c}{$\mathbf{2 0 2 0}$} \\
Months & \multicolumn{2}{c}{$\begin{array}{c}\text { Temperature } \\
\left({ }^{\circ} \mathbf{C}\right)\end{array}$} & \multicolumn{2}{c}{$\begin{array}{c}\text { Humidity } \\
(\%)\end{array}$} & \multicolumn{2}{c}{$\begin{array}{c}\text { Temperature } \\
\left({ }^{\circ} \mathbf{C}\right)\end{array}$} & \multicolumn{2}{c}{$\begin{array}{c}\text { Humidity } \\
(\%)\end{array}$} & \multicolumn{2}{c}{$\begin{array}{c}\text { Temperature } \\
\left({ }^{\circ} \mathbf{C}\right)\end{array}$} & \multicolumn{2}{c}{$\begin{array}{c}\text { Humidity } \\
(\%)\end{array}$} \\
& Max. & Min. & Max. & Min. & Max. & Min. & Max. & Min. & Max. & Min. & Max. & Min. \\
\hline Jan. & 20.2 & 7.4 & 90.1 & 39.3 & 21.0 & 4.1 & 86.9 & 41.2 & 23.2 & 7.1 & 87.5 & 41.0 \\
Feb. & 24.4 & 8.2 & 82.3 & 37.7 & 23.4 & 6.7 & 78.4 & 36.7 & 26.5 & 8.2 & 85.1 & 37.6 \\
Mar. & 28.6 & 9.3 & 74.8 & 28.2 & 26.0 & 8.6 & 83.4 & 34.2 & 30.0 & 11.6 & 79.4 & 29.2 \\
Apr. & 32.2 & 13.7 & 70.8 & 26.4 & 33.1 & 14.3 & 69.4 & 21.5 & 31.5 & 13.6 & 72.2 & 26.9 \\
May. & 35.9 & 18.2 & 59.4 & 19.9 & 37.9 & 19.0 & 58.5 & 19.3 & 39.2 & 20.0 & 57.8 & 18.4 \\
Jun. & 38.7 & 21.9 & 56.6 & 19.5 & 53.3 & 22.4 & 60.3 & 19.6 & 40.4 & 22.6 & 56.8 & 22.0 \\
Jul. & 40.4 & 23.4 & 61.3 & 19.8 & 40.4 & 24.2 & 64.9 & 24.7 & 37.1 & 23.2 & 73.2 & 29.5 \\
Aug. & 38.1 & 23.0 & 65.5 & 27.1 & 39.0 & 22.8 & 69.4 & 27.8 & 38.2 & 22.3 & 69.2 & 29.5 \\
Sep. & 33.1 & 19.7 & 68.0 & 27.9 & 36.3 & 20.3 & 77.7 & 31.9 & 36.9 & 20.9 & 78.1 & 32.3 \\
Oct. & 34.1 & 18.5 & 72.7 & 29.6 & 36.2 & 18.0 & 77.5 & 32.2 & 33.1 & 25.5 & 79.3 & 33.2 \\
Nov. & 26.2 & 10.2 & 82.4 & 35.5 & 29.8 & 14.2 & 83.5 & 38.6 & 29.4 & 14.3 & 82.4 & 39.2 \\
Dec. & 22.8 & 9.0 & 91.1 & 41.4 & 23.4 & 8.0 & 92.1 & 45.0 & 23.7 & 8.3 & 84.7 & 42.7 \\
\hline
\end{tabular}

Table 2. Physical and chemical properties of the soil used at the beginning of the experiment (average of both seasons).

\begin{tabular}{|c|c|c|c|c|c|c|c|c|c|c|c|c|c|c|c|c|c|c|}
\hline & $\begin{array}{l}\text { Partic } \\
\text { distril }\end{array}$ & $\begin{array}{l}\text { le size } \\
\text { ution }\end{array}$ & & 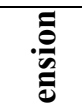 & & & $e^{\circ}$ & & Solu & ble is & ons (m & leq/l, s & oil pa & aste) & & & & \\
\hline & & & $\cong$ & $\frac{\bar{n}}{\bar{n}}$ & 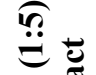 & 0 & $\stackrel{\bar{\Xi}}{\Xi}$ & & Ani & ons & & & Cat & ions & & $\stackrel{\varrho}{e}$ & $\stackrel{e}{e}$ & $e^{o}$ \\
\hline Dू & $\bar{n}$ & U & 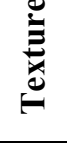 & 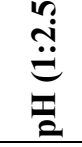 & $ن_{I}^{0}$ & 吾 & 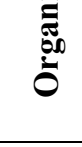 & U & 0 & 巳 & $\overbrace{0}^{+}$ & ש̃ & ${ }^{+50}$ & ${ }^{+\pi}$ & t & $\ddot{\theta}$ & $\stackrel{\theta}{e}$ & ? \\
\hline 23.5 & 27.0 & 49.5 & 离 & 7.71 & 1.13 & 1.85 & 1.87 & 3.52 & - & 4.74 & 3.05 & 5.10 & 0.62 & 1.40 & 4.09 & 0.85 & 0.31 & 0.31 \\
\hline
\end{tabular}

Intermediate Chemicals [NCIC], Egypt) and sowing dates as well as their interaction on growth, fruit yield and essential oil productivity of coriander plants. Coriander seeds were obtained from Agricultural Research Center, El-Dokki, Giza, Egypt.

The experiment consisted of 36 plots in three replicates $\left(3\right.$ sowing dates $\times 4 \mathrm{KH}_{2} \mathrm{PO}_{4}$ concentrations $\times 3$ replicates) consisting of split-plot design; the main plots were sowing date and the sub-plots were potassium dihydrogen orthophosphate concentrations. Each plot was $2 \mathrm{~m}^{2}$ and contained 3 rows with 36 plants, the distance between hills was $25 \mathrm{~cm}$, and each hill contained 2 plants.
The seeds of coriander plants were sown in three different interval dates which were $15^{\text {th }}$ October, $1^{\text {st }}$ November and $15^{\text {th }}$ November for the two experimental seasons. Plots were irrigated directly after planting and after that, they were irrigated regularly at 15-day intervals.

Each experimental unit received the correspondent foliar application of potassium dihydrogen orthophosphate including the tap water (control), 1000, 2000, 3000 and 4000 $\mathrm{ppm}$. The foliar application of the different treatments started 45 days after sowing at the rate of 5 liters from the correspondent treatment per experimental plot divided into 


\section{E.Y. Abdul-Hafeez}

4 repeated times at two-weeks interval. Routine agricultural practices were carried out as usually practiced in coriander cultivation unless otherwise stated. Samples were selected randomly from plants of each plot and data were recorded as follows:

All coriander plants were harvested at the beginning of May when fruits became sufficiently hard. Ten random samples were taken from plants of the middle of the plot and data were recorded on plant height $(\mathrm{cm})$, branch number/plant, stem diameter $(\mathrm{mm})$, umbel number/plant, fruit dry weight/plant $(\mathrm{g})$, fruit dry weight/fed $(\mathrm{kg})$, essential oil \%, essential oil yield/plant (ml) and essential oil yield/fed (liter). Harvest index was calculated by the following formula (A.O.A.C., 1990):

$$
\mathrm{HI}=\frac{\mathrm{FY}}{\mathrm{BY}} \times 100
$$

Where, $\mathrm{HI}=$ harvest index (\%), FY = fruit yield $(\mathrm{kg})$ and $\mathrm{BY}=$ biological yield $=$ dry weight of whole plant $(\mathrm{kg})$.

Dried samples of coriander (100 g) were subjected to hydro-distillation for 3 hours using the Clevenger apparatus for essential oils extraction (Clevenger, 1928) in which water is heated to produce steam, which carries the most volatile chemicals and aromatic material. Essential oil is usually floated on the surface hydrosol (a component of distilled water). Extracted essential oil is stored in a clean Eppendorf glass, in the dark at $4{ }^{\circ} \mathrm{C}$. Essential oil yield in the dried fruits/plant and essential oil yield/fed were calculated.

Data obtained were subjected to the statistical analysis using the "F" Test (Snedecor and Cochran, 1989) and L.S.D. values for the comparison between means of the different treatments according to Steel and Torrie (1982). Statistical analysis was performed using Statistix 8.1 program

\section{RESULTS AND DISCUSSION}

Data presented in Tables (3, 4 and 5) show that sowing date had a significant influence on plant height, branch number/plant, stem diameter, umbel number/plant, fruit dry weight/plant (g), fruit dry weight/fed $(\mathrm{kg})$, essential oil percentage, essential oil yield/plant $(\mathrm{ml})$ and essential oil yield/fed (1). Meanwhile, the harvest index percentage showed no significant response to the different sowing dates during both seasons. Among the three sowing dates tested, plants sown on $1^{\text {st }}$ November recorded the best results regarding all studied characteristics of coriander plants during both seasons, except for plant height during the second season only and stem diameter during both seasons which were significantly increased for the plants sown on $15^{\text {th }}$ October. Plants grown on $15^{\text {th }}$ October significantly surpassed those grown on $15^{\text {th }}$ November in almost all parameters.

The data reveal that plants grown on $1^{\text {st }}$ November reached $111.96 \mathrm{~cm}$ height in the first season and were characterized by more branches (9.39 and 9.48 during both seasons, respectively). Umbel number/plant was significantly improved by early sowing in November (33.46 and 33.23 during both seasons, respectively). Plants grown on $1^{\text {st }}$ November produced significantly higher fruit dw/plant (37.73 and $36.60 \mathrm{~g}$ ) and fruit $\mathrm{dw} /$ fed $(1131.90$ and $1097.90 \mathrm{~kg})$, in both seasons, respectively. A similar effect was noticed in essential oil productivity parameters as inferred from essential oil percentage, essential oil yield per plant and essential oil yield per fed, in both seasons.

Date of sowing is an important management factor for almost all seed spices including coriander. Changing in sowing time leads to a significant change in weather microclimate (Fig., 1) and subsequently the performance of the crop. In addition, the physical environment has a profound influence on growth, biomass partitioning and ultimately the yield of coriander. Temperature, humidity and other meteorological factors may individually or collectively limit the plant growth and productivities. Time of sowing controls the crop phonological development along with the efficient conversion of biomass into 
Table 3. Effect of different sowing dates and foliar applications of potassium dihydrogen orthophosphate on plant height, branch number/plant and stem diameter of coriander during 2018/2019 and 2019/2020 seasons.

\begin{tabular}{|c|c|c|c|c|c|c|c|}
\hline \multirow{2}{*}{$\begin{array}{l}\text { Sowing } \\
\text { dates }\end{array}$} & \multirow{2}{*}{$\begin{array}{l}\mathrm{KH}_{2} \mathrm{PO}_{4} \\
(\mathrm{ppm})\end{array}$} & \multicolumn{2}{|c|}{ Plant height (cm) } & \multicolumn{2}{|c|}{ Branch number/plant } & \multicolumn{2}{|c|}{ Stem diameter (mm) } \\
\hline & & 2018/2019 & $2019 / 2020$ & $2018 / 2019$ & $2019 / 2020$ & 2018/2019 & $2019 / 2020$ \\
\hline & Control & 89.92 & 91.72 & 5.96 & 6.06 & 6.88 & 6.95 \\
\hline & 1000 & 110.98 & 113.20 & 6.37 & 6.48 & 7.19 & 7.26 \\
\hline \multirow[t]{3}{*}{$15^{\text {th }}$ October } & 2000 & 117.01 & 119.35 & 6.44 & 6.55 & 7.37 & 7.44 \\
\hline & 3000 & 119.32 & 121.71 & 6.78 & 6.90 & 7.94 & 8.02 \\
\hline & 4000 & 121.40 & 123.83 & 6.81 & 6.93 & 8.12 & 8.20 \\
\hline \multirow[t]{3}{*}{ Mean } & & 111.73 & 113.96 & 6.47 & 6.58 & 7.50 & 7.58 \\
\hline & Control & 99.15 & 97.16 & 9.08 & 9.17 & 6.86 & 6.98 \\
\hline & 1000 & 113.77 & 111.34 & 9.15 & 9.24 & 6.91 & 7.03 \\
\hline \multirow[t]{3}{*}{$1^{\text {st }}$ November } & 2000 & 113.61 & 111.55 & 9.20 & 9.29 & 7.10 & 7.22 \\
\hline & 3000 & 116.29 & 113.97 & 9.73 & 9.83 & 7.29 & 7.41 \\
\hline & 4000 & 117.00 & 114.66 & 9.81 & 9.91 & 7.55 & 7.68 \\
\hline \multirow[t]{3}{*}{ Mean } & & 111.96 & 109.72 & 9.39 & 9.48 & 7.14 & 7.26 \\
\hline & Control & 80.67 & 83.09 & 4.86 & 4.91 & 3.98 & 3.96 \\
\hline & 1000 & 84.17 & 86.69 & 5.24 & 5.29 & 4.13 & 4.11 \\
\hline \multirow[t]{3}{*}{$15^{\text {th }}$ November } & 2000 & 84.38 & 86.91 & 5.63 & 5.69 & 4.29 & 4.26 \\
\hline & 3000 & 87.67 & 90.30 & 6.05 & 6.11 & 4.39 & 4.36 \\
\hline & 4000 & 93.30 & 96.10 & 6.15 & 6.21 & 4.53 & 4.50 \\
\hline \multirow[t]{2}{*}{ Mean } & & 86.04 & 88.62 & 5.59 & 5.64 & 4.26 & 4.24 \\
\hline & Control & 89.91 & 90.66 & 6.64 & 6.71 & 5.91 & 5.96 \\
\hline \multirow{4}{*}{$\begin{array}{l}\text { Means of } \\
\mathrm{KH}_{2} \mathrm{PO}_{4} \\
\text { concentrations }\end{array}$} & 1000 & 102.97 & 103.80 & 6.92 & 7.00 & 6.07 & 6.13 \\
\hline & 2000 & 105.00 & 105.86 & 7.09 & 7.18 & 6.25 & 6.31 \\
\hline & 3000 & 107.76 & 108.66 & 7.52 & 7.61 & 6.53 & 6.60 \\
\hline & 4000 & 110.57 & 111.53 & 7.59 & 7.68 & 6.73 & 6.79 \\
\hline \multirow[t]{3}{*}{ LSD 0.05} & Sowing dates & 2.55 & 2.59 & 0.12 & 0.36 & 0.03 & 0.18 \\
\hline & $\mathrm{KH}_{2} \mathrm{PO}_{4}$ & 2.24 & 2.27 & 0.09 & 0.09 & 0.06 & 0.06 \\
\hline & Interaction & 3.88 & 3.94 & 0.16 & 0.16 & 0.11 & 0.10 \\
\hline
\end{tabular}

\footnotetext{
* LSD values to compare the means under the same level of sowing date, and values between parentheses
} to compare the means under different levels of sowing date. 
Table 4. Effect of different sowing dates and foliar applications of potassium dihydrogen orthophosphate on umbel number/plant, fruit dry weight/plant, fruit dry weight/fed and harvest index of coriander during 2018/2019 and 2019/2020 seasons.

\begin{tabular}{|c|c|c|c|c|c|c|c|c|c|}
\hline \multirow{2}{*}{$\begin{array}{l}\text { Sowing } \\
\text { dates }\end{array}$} & \multirow{2}{*}{$\begin{array}{l}\mathrm{KH}_{2} \mathrm{PO}_{4} \\
(\mathrm{ppm})\end{array}$} & \multicolumn{2}{|c|}{$\begin{array}{c}\text { Umbel } \\
\text { number/plant }\end{array}$} & \multicolumn{2}{|c|}{$\begin{array}{c}\text { Fruit DW/plant } \\
\text { (g) }\end{array}$} & \multicolumn{2}{|c|}{ Fruit dw/fed (kg) } & \multicolumn{2}{|c|}{ Harvest index (\%) } \\
\hline & & $\begin{array}{l}2018 / \\
2019 \\
\end{array}$ & $\begin{array}{l}2019 / \\
2020 \\
\end{array}$ & $\begin{array}{l}2018 / \\
2019 \\
\end{array}$ & $\begin{array}{l}2019 / \\
2020\end{array}$ & $\begin{array}{l}2018 / \\
2019 \\
\end{array}$ & $\begin{array}{l}2019 / \\
2020 \\
\end{array}$ & $\begin{array}{l}2018 / \\
2019\end{array}$ & $\begin{array}{l}2019 / \\
2020 \\
\end{array}$ \\
\hline \multirow{5}{*}{$15^{\text {th }}$ October } & Control & 25.26 & 24.92 & 26.38 & 26.02 & 791.50 & 780.72 & 58.15 & 57.50 \\
\hline & 1000 & 26.90 & 26.54 & 29.18 & 28.80 & 875.30 & 863.88 & 61.87 & 61.14 \\
\hline & 2000 & 26.87 & 26.51 & 30.52 & 30.12 & 915.50 & 903.49 & 67.29 & 66.47 \\
\hline & 3000 & 27.88 & 27.51 & 32.61 & 32.17 & 978.20 & 964.98 & 71.97 & 70.98 \\
\hline & 4000 & 28.87 & 28.49 & 34.10 & 33.65 & 1023.00 & 1009.59 & 75.54 & 74.55 \\
\hline \multirow[t]{3}{*}{ Mean } & & 27.16 & 26.79 & 30.56 & 30.15 & 916.80 & 904.50 & 66.96 & 66.13 \\
\hline & Control & 22.90 & 22.73 & 31.83 & 30.88 & 955.00 & 926.35 & 68.74 & 66.68 \\
\hline & 1000 & 34.67 & 34.43 & 35.60 & 34.53 & 1068.00 & 1035.96 & 74.44 & 72.21 \\
\hline \multirow[t]{3}{*}{$1^{\text {st }}$ November } & 2000 & 35.27 & 35.04 & 39.03 & 37.86 & 1171.00 & 1135.87 & 79.94 & 77.54 \\
\hline & 3000 & 36.67 & 36.43 & 39.87 & 38.67 & 1196.00 & 1160.12 & 85.53 & 82.97 \\
\hline & 4000 & 37.79 & 37.54 & 42.31 & 41.04 & 1269.33 & 1231.25 & 98.24 & 95.29 \\
\hline \multirow[t]{3}{*}{ Mean } & & 33.46 & 33.23 & 37.73 & 36.60 & 1131.90 & 1097.90 & 81.38 & 78.94 \\
\hline & Control & 12.70 & 13.00 & 27.47 & 28.11 & 824.00 & 843.32 & 57.67 & 59.06 \\
\hline & 1000 & 13.24 & 13.55 & 28.30 & 28.96 & 849.00 & 868.83 & 58.50 & 59.93 \\
\hline \multirow[t]{3}{*}{$15^{\text {th }}$ November } & 2000 & 13.72 & 14.04 & 29.19 & 29.86 & 875.60 & 895.91 & 63.58 & 65.03 \\
\hline & 3000 & 14.22 & 14.55 & 30.92 & 31.64 & 927.50 & 949.19 & 68.60 & 70.25 \\
\hline & 4000 & 14.74 & 15.08 & 33.06 & 33.82 & 991.70 & 1014.72 & 75.41 & 77.16 \\
\hline \multirow[t]{2}{*}{ Mean } & & 13.72 & 14.04 & 29.79 & 30.48 & 893.60 & 914.30 & 64.75 & 66.29 \\
\hline & Control & 20.28 & 20.22 & 28.56 & 28.34 & 856.90 & 850.10 & 61.52 & 61.08 \\
\hline \multirow{4}{*}{$\begin{array}{l}\text { Means of } \\
\mathrm{KH}_{2} \mathrm{PO}_{4} \\
\text { concentrations }\end{array}$} & 1000 & 24.94 & 24.84 & 31.03 & 30.76 & 930.80 & 922.80 & 64.94 & 64.43 \\
\hline & 2000 & 25.27 & 25.20 & 32.91 & 32.61 & 987.40 & 978.30 & 70.27 & 69.68 \\
\hline & 3000 & 26.26 & 26.16 & 34.46 & 34.16 & 1034.00 & 1024.90 & 75.37 & 74.73 \\
\hline & 4000 & 27.13 & 27.04 & 36.49 & 36.17 & 1094.70 & 1085.20 & 83.06 & 82.33 \\
\hline \multirow[t]{3}{*}{ LSD 0.05} & Sowing dates & 1.36 & 1.67 & 1.29 & 1.61 & 38.93 & 48.46 & N.S. & N.S. \\
\hline & $\mathrm{KH}_{2} \mathrm{PO}_{4}$ & 1.73 & 1.72 & 0.72 & 0.71 & 21.67 & 21.33 & 11.14 & 10.90 \\
\hline & Interaction & 2.99 & 2.98 & 1.25 & 1.24 & 37.55 & 36.95 & N.S. & N.S. \\
\hline
\end{tabular}

* LSD values to compare the means under the same level of sowing date, and values between parentheses to compare the means under different levels of sowing date. NS denotes non-significant differences at $p=0.05$ by LSD. 
Table 5. Effect of different sowing dates and foliar applications of potassium dihydrogen orthophosphate on essential oil percentage, essential oil yield/plant and essential oil yield/fed of coriander during 2018/2019 and 2019/2020 seasons.

\begin{tabular}{|c|c|c|c|c|c|c|c|}
\hline \multirow{2}{*}{$\begin{array}{l}\text { Sowing } \\
\text { dates }\end{array}$} & \multirow{2}{*}{$\begin{array}{l}\mathrm{KH}_{2} \mathrm{PO}_{4} \\
(\mathbf{p p m})\end{array}$} & \multicolumn{2}{|c|}{$\begin{array}{c}\text { Essential oil } \\
(\%)\end{array}$} & \multicolumn{2}{|c|}{$\begin{array}{l}\text { Essential oil yield/plant } \\
\text { (ml) }\end{array}$} & \multicolumn{2}{|c|}{$\begin{array}{l}\text { Essential oil yield/fed } \\
\text { (liter) }\end{array}$} \\
\hline & & 2018/2019 & $2019 / 2020$ & 2018/2019 & $2019 / 2020$ & 2018/2019 & $2019 / 2020$ \\
\hline \multirow{5}{*}{$15^{\text {th }}$ October } & Control & 2.03 & 2.02 & 0.54 & 0.53 & 16.11 & 15.78 \\
\hline & 1000 & 2.37 & 2.35 & 0.69 & 0.68 & 20.72 & 20.33 \\
\hline & 2000 & 2.53 & 2.52 & 0.77 & 0.76 & 23.20 & 22.76 \\
\hline & 3000 & 2.77 & 2.75 & 0.90 & 0.88 & 27.05 & 26.55 \\
\hline & 4000 & 2.80 & 2.78 & 0.95 & 0.94 & 28.64 & 28.06 \\
\hline \multirow[t]{3}{*}{ Mean } & & 2.50 & 2.48 & 0.77 & 0.76 & 23.14 & 22.70 \\
\hline & Control & 2.23 & 2.26 & 0.71 & 0.70 & 21.32 & 20.90 \\
\hline & 1000 & 2.57 & 2.59 & 0.91 & 0.90 & 27.43 & 26.89 \\
\hline \multirow[t]{3}{*}{$1^{\text {st }}$ November } & 2000 & 2.70 & 2.73 & 1.05 & 1.03 & 31.63 & 31.01 \\
\hline & 3000 & 2.87 & 2.90 & 1.14 & 1.12 & 34.27 & 33.57 \\
\hline & 4000 & 3.17 & 3.20 & 1.34 & 1.31 & 40.21 & 39.42 \\
\hline \multirow[t]{3}{*}{ Mean } & & 2.71 & 2.74 & 1.03 & 1.01 & 30.97 & 30.36 \\
\hline & Control & 2.10 & 2.13 & 0.58 & 0.60 & 17.30 & 17.93 \\
\hline & 1000 & 2.20 & 2.23 & 0.62 & 0.65 & 18.67 & 19.36 \\
\hline \multirow[t]{3}{*}{$15^{\text {th }}$ November } & 2000 & 2.30 & 2.33 & 0.67 & 0.70 & 20.15 & 20.89 \\
\hline & 3000 & 2.43 & 2.47 & 0.75 & 0.78 & 22.56 & 23.39 \\
\hline & 4000 & 2.60 & 2.64 & 0.86 & 0.89 & 25.80 & 26.74 \\
\hline \multirow[t]{2}{*}{ Mean } & & 2.33 & 2.35 & 0.70 & 0.72 & 20.90 & 21.66 \\
\hline & Control & 2.12 & 2.14 & 0.61 & 0.60 & 18.24 & 18.20 \\
\hline \multirow{4}{*}{$\begin{array}{l}\text { Means of } \\
\mathrm{KH}_{2} \mathrm{PO}_{4} \\
\text { concentrations }\end{array}$} & 1000 & 2.38 & 2.39 & 0.74 & 0.74 & 22.28 & 22.19 \\
\hline & 2000 & 2.51 & 2.53 & 0.83 & 0.82 & 24.99 & 24.88 \\
\hline & 3000 & 2.69 & 2.70 & 0.93 & 0.92 & 27.96 & 27.84 \\
\hline & 4000 & 2.86 & 2.87 & 1.05 & 1.04 & 31.54 & 31.41 \\
\hline \multirow[t]{3}{*}{ LSD 0.05} & Sowing dates & 0.12 & 0.15 & 0.04 & 0.04 & 1.14 & 1.21 \\
\hline & $\mathrm{KH}_{2} \mathrm{PO}_{4}$ & 0.09 & 0.09 & 0.04 & 0.03 & 1.07 & 1.06 \\
\hline & Interaction & 0.16 & 0.16 & 0.06 & 0.06 & 1.86 & 1.85 \\
\hline
\end{tabular}

\footnotetext{
* LSD values to compare the means under the same level of sowing date, and values between parentheses
} to compare the means under different levels of sowing date. 


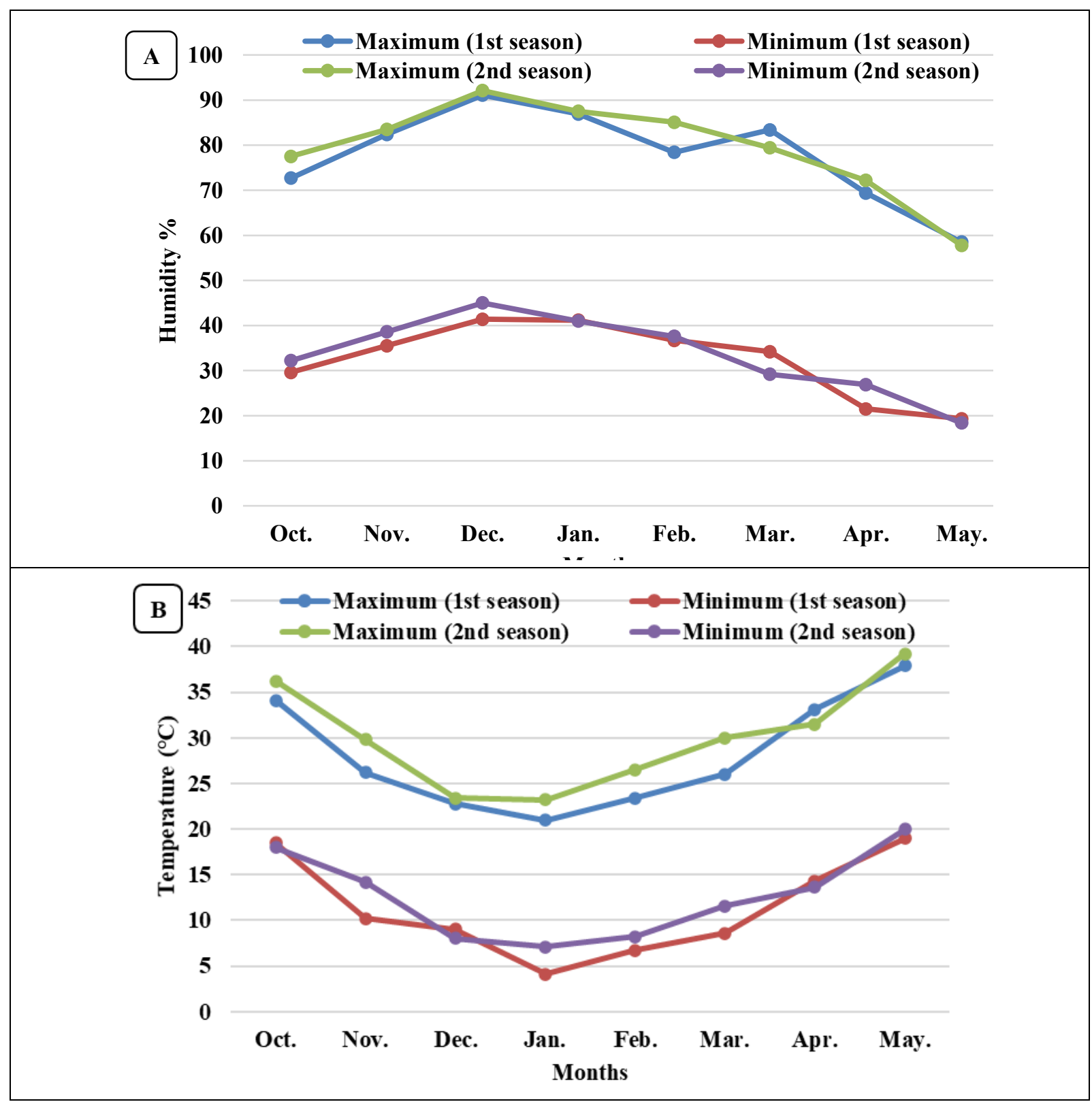

Fig. 1. Meteorological data pertaining to temperature (A) and relative humidity (B).

economic yield (Khichar and Niwas, 2006). In an investigation on the influence of sowing date, Hornek (1976) observed that low temperature at the time of emergence caused slow germination of coriander. Plant height, number of branches and fresh weight of leaves were maximum in $15^{\text {th }}$ October and $1^{\text {st }}$ November sown crop and this was due to the favorable agro-climatic conditions. The present study is in conformity with the findings of Naghera et al. (2000) and Tiwari et al. (2002) in coriander.
The correlation matrix (Table, 6) revealed significant correlation coefficient values at $\mathrm{p} \leq 0.05$ and $\mathrm{p} \leq 0.01$ among most coriander growth characteristics, fruit yield and essential oil productivity as affected by sowing date treatments. Among the most obvious significant correlation is that between fruit dry weight/plant and essential oil yield/plant $(\mathrm{r}=0.97$ and 0.96$)$, in both seasons, respectively. Umbel number/plant was significantly correlated with fruit DW/plant $(\mathrm{r}=0.81$ and 0.74$)$ and essential oil yield/plant $(\mathrm{r}=0.78$ and 0.73$)$ in both seasons, 
Table 6. Correlation coefficients matrix (r) of growth characteristics of Coriandrum sativum $L$. plants as affected by the different sowing dates during 2018/2019 $\left(1^{\text {st }}\right)$ and $2019 / 2020\left(2^{\text {nd }}\right)$ seasons.

\begin{tabular}{|c|c|c|c|c|c|c|c|c|c|c|}
\hline Characteristics & 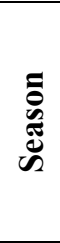 & 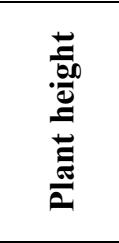 & 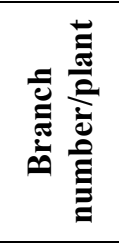 & 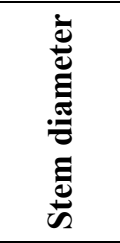 & 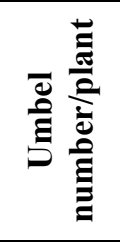 & 咅 & 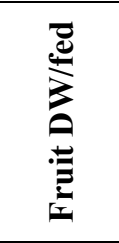 & 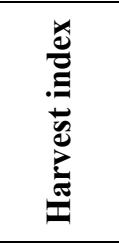 & 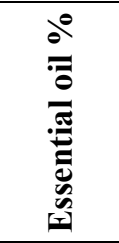 & 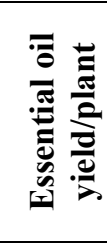 \\
\hline \multirow{2}{*}{ Branch number/plant } & $1^{\text {st }}$ & $0.78 * *$ & - & - & - & - & - & - & - & - \\
\hline & $2^{\text {nd }}$ & $0.70 * *$ & - & - & - & - & - & - & - & - \\
\hline \multirow{2}{*}{ Stem diameter } & $1^{\text {st }}$ & $0.93 * *$ & $0.77 * *$ & - & - & - & - & - & - & - \\
\hline & $2^{\text {nd }}$ & $0.90 * *$ & $0.78 * *$ & - & - & - & - & - & - & - \\
\hline \multirow{2}{*}{ Umbel number/plant } & $1^{\text {st }}$ & $0.91 * *$ & $0.89 * *$ & $0.90 * *$ & - & - & - & - & - & - \\
\hline & $2^{\text {nd }}$ & $0.86^{* *}$ & $0.89 * *$ & $0.90 * *$ & - & - & - & - & - & - \\
\hline \multirow{2}{*}{ Fruit DW/plant } & $1^{\text {st }}$ & $0.75 * *$ & $0.87 * *$ & $0.63 * *$ & $0.81 * *$ & - & - & - & - & - \\
\hline & $2^{\text {nd }}$ & $0.64 * *$ & $0.80 * *$ & $0.54 *$ & $0.74 * *$ & - & - & - & - & - \\
\hline \multirow{2}{*}{ Fruit DW/fed } & $1^{\text {st }}$ & $075 * *$ & $0.87 * *$ & $0.63 * *$ & $0.81 * *$ & $1.00 * *$ & - & - & - & - \\
\hline & $2^{\text {nd }}$ & $0.64 * *$ & $0.80 * *$ & $0.54 *$ & $0.74 * *$ & $1.00 * *$ & - & - & - & - \\
\hline \multirow{2}{*}{ Harvest index } & $1^{\text {st }}$ & $0.57 *$ & $0.62 * *$ & 0.46 & $0.58 *$ & $0.74 * *$ & $0.74 * *$ & - & - & - \\
\hline & $2^{\text {nd }}$ & 0.47 & $0.55^{*}$ & 0.37 & $0.50 *$ & $0.71 * *$ & $0.71 * *$ & - & - & - \\
\hline \multirow{2}{*}{ Essential oil \% } & $1^{\text {st }}$ & $0.74 * *$ & $0.66 * *$ & $0.59 * *$ & $0.69 * *$ & $0.85^{* *}$ & $0.85 * *$ & $0.69 * *$ & - & - \\
\hline & $2^{\text {nd }}$ & $0.70 * *$ & $0.63 * *$ & $0.54 *$ & $0.66 * *$ & $0.85 * *$ & $0.85 * *$ & $0.63 * *$ & - & - \\
\hline \multirow{2}{*}{ Essential oil yield/plant } & $1^{\text {st }}$ & $0.76^{* *}$ & $0.80 * *$ & $0.63 * *$ & $0.78 * *$ & $0.97 * *$ & $0.96^{* *}$ & $0.75^{* *}$ & $0.95 * *$ & - \\
\hline & $2^{\text {nd }}$ & $0.68 * *$ & $0.74 * *$ & $0.56^{*}$ & $0.73 * *$ & $0.96 * *$ & $0.96^{* *}$ & $0.69 * *$ & $0.96 * *$ & - \\
\hline \multirow{2}{*}{ Essential oil yield/fed } & $1^{\text {st }}$ & $0.76^{* *}$ & $0.80 * *$ & $0.63 * *$ & $0.78 * *$ & $0.97 * *$ & $0.96 * *$ & $0.74 * *$ & $0.95 * *$ & $0.99 * *$ \\
\hline & $2^{\text {nd }}$ & $0.68 * *$ & $0.74 * *$ & $0.56^{*}$ & $0.73 * *$ & $0.96^{* *}$ & $0.96^{* *}$ & $0.69 * *$ & $0.96 * *$ & $0.99 * *$ \\
\hline
\end{tabular}

* Correlation is significant at the $5 \%$ level.

** Correlation is significant at the $1 \%$ level.

respectively. Meanwhile, stem diameter slightly and non-significantly correlated with the harvest index in both seasons $(r=0.46$ and 0.37 ).

Treating coriander plants with the foliar application of potassium dihydrogen orthophosphate exhibited a significant effect on plant height, branch number/plant, stem diameter, umbel number/plant, fruit dry weight per plant and per fed, harvest index, essential oil percentage and essential oil yield per plant and per fed. Although plant height significantly differed according to the different concentrations of potassium dihydrogen orthophosphate treatment. The high concentration at $4000 \mathrm{ppm}$ showed the best results of all studied parameters comparing to control and surpassed the other concentrations during both seasons. No significant effects were recorded between the highest concentration at $3000 \mathrm{ppm}$ and the next concentration at $4000 \mathrm{ppm}$ for the plant parameters of branch number/plant, umbels number/plant and harvest index in both seasons. Harvest index offers an indication about the sharing of photosynthesis between the different parts of the plant, therefore it represents the measure of the biological efficiency and production efficiency of the crop.

Potassium fertilization has important influences on the contents of harvested crops and the quality of diet, especially under stress (Ashraf et al., 2013). However, potassium content in soils is usually limited, and so the plant yields are restricted. The evidence of the diverse functions of potassium from the molecular level to field performance had been increased. The role of potassium to reduce drought and salinity stress is becoming very important (Cakmak, 2005; Amtmann et al., 2008; Wang and Wu, 2010). In our investigation, adding potassium was very helpful for coriander 
plant growth and essential oil productivity, which resulted from the fact that potassium fertilizer promoted root growth. The same result on soybean was obtained by Fernández et al. (2009). Tripathi et al. (2009) recorded that the fruit yield of coriander increased with the fertilization of potassium and sulfur.

Potassium is highly mobile element in plants and contents up to 10 percent of plant dry weight (Adams and Shin, 2014; Shin, 2014 and Walker et al., 1996). Depending on the total amount of mineral nutrients required by plants, potassium element is required with a large amount after nitrogen element (Zörb et al., 2014). Moreover, it is the largest element required by the fruits (Lester et al., 2006; Mpelasoka et al., 2003). Potassium activates some enzymes, which are critical for the different metabolic processes, like biosynthesis, transformation and transport of sugar and starch (Karley and White, 2009; Niu et al., 2013; Römheld and
Kirkby, 2010). In addition, potassium is an important nutrient involved in the phloem translocation of assimilates (Lebaudy et al., 2007). Generally, it is an important element, which could enhance fruit development and produce high quality fruits and longer shelf life by increasing the synthesis and translocation of carbohydrates in plants (Niu et al., 2008). All previous studies authenticate with our results including the enhancement in the growth, fruit yield and essential oil production through the foliar application of potassium at a high concentration rate.

The correlation matrix presented in Table (7) revealed significant correlation coefficient values at $\mathrm{p} \leq 0.05$ and $\mathrm{p} \leq 0.01$ among most coriander growth characteristics, yield and essential oil content as affected by potassium dihydrogen orthophosphate applications.

Table 7. Correlation coefficients matrix (r) of growth characteristics of Coriandrum sativum $\mathrm{L}$. plants as affected by the foliar application of potassium dihydrogen orthophosphate at different concentrations during 2018/2019 (1 $\left.{ }^{\text {st }}\right)$ and $2019 / 2020\left(2^{\text {nd }}\right)$ seasons.

\begin{tabular}{|c|c|c|c|c|c|c|c|c|c|c|}
\hline Characteristics & 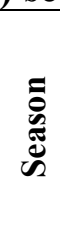 & 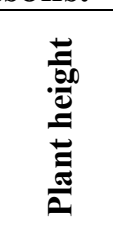 & 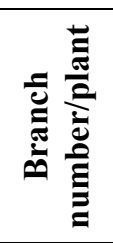 & 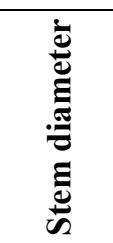 & 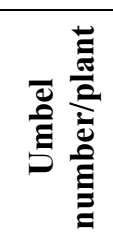 & 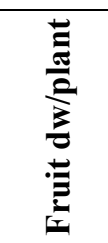 & 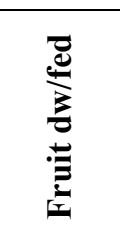 & 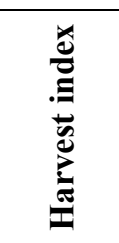 & $\frac{\partial}{0}$ & 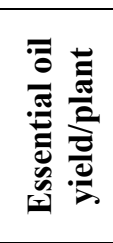 \\
\hline \multirow{2}{*}{ Branch number/plant } & $1^{\text {st }}$ & $0.61 * *$ & - & - & - & - & - & - & - & - \\
\hline & $2^{\text {nd }}$ & 0.49 & - & - & - & - & - & - & - & - \\
\hline \multirow{2}{*}{ Stem diameter } & $1^{\text {st }}$ & $0.95 * *$ & $0.58^{*}$ & - & - & - & - & - & - & - \\
\hline & $2^{\text {nd }}$ & $0.93 * *$ & $0.60 * *$ & - & - & - & - & - & - & - \\
\hline \multirow{2}{*}{ Umbel number/plant } & $1^{\text {st }}$ & $0.86^{* *}$ & $0.87 * *$ & $0.86^{* *}$ & - & - & - & - & - & - \\
\hline & $2^{\text {nd }}$ & $0.77 * *$ & $0.88 * *$ & $0.86^{* *}$ & - & - & - & - & - & - \\
\hline \multirow{2}{*}{ Fruit dw/plant } & $1^{\text {st }}$ & $0.59 *$ & $0.91 * *$ & 0.49 & $0.78 * *$ & - & - & - & - & - \\
\hline & $2^{\text {nd }}$ & 0.41 & $0.85^{* *}$ & 0.40 & $0.71 * *$ & - & - & - & - & - \\
\hline \multirow{2}{*}{ Fruit dw/fed } & $1^{\text {st }}$ & $0.59 *$ & $0.91 * *$ & 0.49 & $0.78 * *$ & $1.00 * *$ & - & - & - & - \\
\hline & $2^{\text {nd }}$ & 0.41 & $0.85^{* *}$ & 0.40 & $0.71 * *$ & $1.00 * *$ & - & - & - & - \\
\hline \multirow{2}{*}{ Harvest index } & $1^{\text {st }}$ & 0.41 & $0.61 * *$ & 0.33 & $0.51 *$ & $0.70 * *$ & $0.69 * *$ & - & - & - \\
\hline & $2^{\text {nd }}$ & 0.29 & $0.55^{*}$ & 0.24 & 0.45 & $0.67 * *$ & $0.67 * *$ & - & - & - \\
\hline \multirow{2}{*}{ Essential oil \% } & $1^{\text {st }}$ & $0.69 * *$ & $0.67 * *$ & $0.59 *$ & $0.69 * *$ & $0.82 * *$ & $0.83 * *$ & $0.62 * *$ & - & - \\
\hline & $2^{\text {nd }}$ & $0.58 *$ & $0.66 * *$ & $0.52 *$ & $0.66^{* *}$ & $0.82 * *$ & $0.82 * *$ & $0.55^{*}$ & - & - \\
\hline \multirow{2}{*}{ Essential oil yield/plant } & $1^{\text {st }}$ & $0.64 * *$ & $0.84 * *$ & $0.55^{*}$ & $0.78 * *$ & $0.96 * *$ & $0.96 * *$ & $0.69 * *$ & $0.94 * *$ & - \\
\hline & $2^{\text {nd }}$ & $0.50 *$ & $0.80 * *$ & 0.48 & $0.72 * *$ & $0.95 * *$ & $0.95 * *$ & $0.63 * *$ & $0.95 * *$ & - \\
\hline \multirow{2}{*}{ Essential oil yield/fed } & $1^{\text {st }}$ & $0.64 * *$ & $0.84 * *$ & $0.54^{*}$ & $0.78 * *$ & $0.96 * *$ & $0.96 * *$ & $0.69 * *$ & $0.94 * *$ & $0.99 * *$ \\
\hline & $2^{\text {nd }}$ & $0.50 *$ & $0.80 * *$ & 0.48 & $0.72 * *$ & $0.95 * *$ & $0.95 * *$ & $0.63 * *$ & $0.95 * *$ & $0.99 * *$ \\
\hline
\end{tabular}

* Correlation is significant at the $5 \%$ level.

** Correlation is significant at the $1 \%$ level. 
Among the most obvious significant correlations is that between umbel number/plant and each of fruit dw/plant $(\mathrm{r}=0.78$ and 0.71$)$ and essential oil yield/plant $(\mathrm{r}=0.78$ and 0.72$)$, in both seasons, respectively. Branch number/plant was significantly correlated with umbel number/plant $\quad(\mathrm{r}=0.87$ and 0.88), fruit $\mathrm{dw} /$ plant $(\mathrm{r}=0.94$ and 0.85$)$ and essential oil yield/plant ( $\mathrm{r}=0.84$ and 0.80$)$ in both seasons, respectively. Meanwhile, stem diameter slightly and non-significantly correlated with harvest index in both seasons $(r=0.33$ and $0.24)$.

Both foliar application of potassium dihydrogen orthophosphate and sowing date significantly interacted with respect to all characteristics of coriander plants in both seasons except for harvest index percentage. The best results were noticed in the plants cultivated on $1^{\text {st }}$ November and treated with potassium at $4000 \mathrm{ppm}$ in all parameters except for plant height which achieved the best combination when plants were sown on $15^{\text {th }}$ October and treated with potassium at $4000 \mathrm{ppm}$.

\section{CONCLUSION}

Comparatively speaking, the foliar application of $\mathrm{K}_{2} \mathrm{PO}_{4}$ could significantly increase the plant height, branch number, umbel number, fruit dry weight as well as the essential oil percentage and yield. The cultivation of coriander plants cultivated early in November ( $1^{\text {st }}$ November) could significantly enhance most of growth and quality parameters. Farmers should adopt different strategies according to the aim of whether to increase the fruit and essential oil yield or improve the fruit quality.

\section{REFERENCES}

Adams, E. and Shin, R. (2014). Transport, signaling, and homeostasis of potassium and sodium in plants. J. Integr. Plant Biol., 56: 231-249.

Amtmann, A.; Troufflard, S. and Armengaud, P. (2008). The effect of potassium nutrition on pest and disease resistance in plants. Physiol. Plant., 133: 682-691.

A.O.A.C. (1990). Official Methods of Analysis of the Association of Official Analytical Chemists, $15^{\text {th }} \quad$ Ed. Washington D.C., USA. 1298 p.

Ashraf, M.Y.; Rafique, N.; Ashraf, M.; Azhar, N. and Marchand, M. (2013). Effect of supplemental potassium $\left(\mathrm{K}^{+}\right)$on growth, physiological and biochemical attributes of wheat grown under saline conditions. J. Plant Nutr., 36: 443-458.

Awad, M.A.; Ismail, S.M. and Al-Qurashi, A.D. (2014). Effect of potassium soil and foliar spray fertilization on yield, fruit quality and nutrient uptake of 'Seweda' date palm grown in sandy loam soil. Journal of Food, Agriculture \& Environment, 12: 305-311.

Ayanoglue, F.; Ahmet, M.; Neset, A. and Bilal, B. (2002). Seed yields, yield components and essential oil of selected coriander (Coriandrum sativum L.) lines. J. Herbs Spices Med. Plants, 9: 71-76.

Black, C.A.; Evans, D.D.; White, J.I.; Ensminger, L.E. and Clark, F.E. (1982). Methods of Soil Analysis. American Society of Agronomy, Soil Science Society of America, Madison, Wisconsin, USA, $1569 \mathrm{p}$.

Britto, D.T. and Kronzucker, H.J. (2008). Cellular mechanisms of potassium transport in plants. Physiologia Plantarum, 133: 637-650.

Cakmak, I. (2005). The role of potassium in alleviating detrimental effects of abiotic stresses in plants. J. Plant Nutr. Soil Sci., 168: 521-530.

Cao, Y.; Glass, A.D.M. and Crawford, N. M. (1993). Ammonium inhibition of arabidopsis root growth can be reversed by potassium and by auxin resistance mutations auxl, axrl, and axr2. Plant Physiology, 102: 983-989.

Carrubba, A.; Torre, R.; Saiano, F. and Alonzo, G. (2006). Effect of sowing time 


\section{E.Y. Abdul-Hafeez}

on coriander performance in a semi-arid Mediterranean environment. Crop Sci., 46: 437-447.

Chauduni, B.B. and De, R. (1975). Effect of soil and foliar application of phosphorus on yield of tomato (Lycopersicon esculentum Mill.). Soil Science and Plant Nutrition, 21: 57-62.

Clevenger, J.H. (1928). Apparatus for the determination of volatile oil. J. Amer. Pharm. Assoc., 17(4):345-349.

Diederichsen, A. (1996). Coriander, Coriandrum sativum L. International Plant Genetic Resources Institute (IPGRI), Rome, Italy, $83 \mathrm{p}$.

Elgayyar, M.; Draughon, F.A.; Golden, D.A. and Mount, J.A. (2001). Antimicrobial activity of essential oils from plants against selected pathogenic and saprophytic microorganisms. J. Food Protect, 64:1019-1024.

Fan, L. and Moshe, S. (2002). Response of maize to foliar vs soil application of nitrogen-phosphorus-potassium fertilizers. Journal of Plant Nutrition, 25: 23332342.

Fernández, F.G.; Brouder, S.M.; Volenec, J.J.; Beyrouty, C.A. and Hoyum, R. (2009). Root and shoot growth, seed composition, and yield components of no-till rainfed soybean under variable potassium. Plant Soil, 322:125-138.

Gil, A.; De-la-Fuente, E.; Lenardis, A.; Lorenzo, S.; Marengo, J. and De-laFuente, E. (1999). Coriander (Coriandrum sativum L.) yield response to plant populations. J. Herbs Spices Med. Plants, 6 (3):63-73.

Gil, A.; De-La-Fuente, E.B.; Lenardis, A.E.; Pereira, M.L.; Suarez, S.A.; Arnaldo, B.; Van Baren, C.; Di Leo, L.P. and Ghersa, C.M. (2002). Coriander essential oil composition from two genotypes grown in different environmental conditions. J. Agric. Food Chem., 50: 2870-2877.
Giskin, M.L.; Santos, A.T. and Etcheves, J.D. (1984). Can the foliar application of essential nutrients decrease fertilizer inputs? In: Pierre-Martin, P. (ed.), Proceedings of the $7^{\text {th }}$ Colloquium for the Optimalization of Plant Nutrition Vol. I, New York, Springer, pp. 239-242.

Hagin, J.; Olsen, S.R. and Shaviv, A. (1990). Review of interaction of ammoniumnitrate and potassium nutrition of crops. Journal of Plant Nutrition, 13:12111226.

Hornek, L. (1976). The effect of sowing date on the yield and essential oil content of coriander (Coriandrum sativum L.). Jerba Hungarica, 15: 55-62.

Jackson, M.L. (1973). Soil Chemical Analysis. Prentic-Hall. Inc. Englewood, Cliffs, USA, 498 p.

Jeliazkova, E.A.; Craker, L.E. and Zheljazkov, V.D. (1997). Irradiation of seeds and productivity of coriander, Coriandrum sativum L. J. Herbs Spices Med. Plants, 5:73-79.

Karley, A.J. and White, P.J. (2009). Moving cationic minerals to edible tissues: Potassium, magnesium, calcium. Curr. Opin. Plant Biol., 12: 291-298.

Khichar, M.L. and Niwas, R. (2006). Microclimate profiles under different sowing environments in wheat. J. Agromet, 8: 201-209.

Lebaudy, A.; Véry, A.A. and Sentenac, H. (2007). $\mathrm{K}^{+}$channel activity in plants: Genes, regulations and functions. FEBS Lett., 581: 2357-2366.

Lenardis, A.D.; De-la-Fuente, E.; Gil, A. and Tubia, A. (2000). Response of coriander (Coriandrum sativum L.) to nitrogen availability. J. Herbs Spices Med. Plants, 7: 47-58.

Lester, G.E.; Jifon, J.L. and Makus, D.J. (2006). Supplemental foliar potassium applications with or without a surfactant can enhance netted muskmelon quality. HortScience, 41: 741-744. 
Luayza, G.; Brevedan, R. and Palomo, R. (1996). Coriander under irrigation in Argentina. In: Janick, J. (ed.), Progress in New Crops, ASHS Press, Arlington, pp. 590-594.

Machado, A.S.R.; De Azevedo, E.G.; Da Ponte, M.N. and Sardinha, R.M.A. (1993). High pressure carbon dioxide extraction from coriander plants/headspace analysis. J. Essent. Oil Res., 5:645-649.

Mpelasoka, B.S.; Schachtman, D.P.; Treeby, M.T. and Thomas, M.R. (2003). A review of potassium nutrition in grapevines with special emphasis on berry accumulation. Austral. J. Grape Wine Res., 9: 154-168.

Naghera, R.P.; Sukhadia, H.M. and Ramani, B.B. (2000). Effect of sowing dates and varying levels of nitrogen and phosphorus of coriander (Coriandrum sativum L.). Gujarat Agric. Univ. Res. J., 26:52-54.

Niu, J.F.; Zhang, W.F.; Ru, S.H.; Chen, X.P.; Xiao, K.; Zhang, X.Y.; Assaraf, M.; Imas, P.; Magen, H. and Zhang, F.S. (2013). Effects of potassium fertilization on winter wheat under different production practices in the North China Plain. Field Crops Res., 140: 69-76.

Niu, Z.M.; Xu, X.F.; Wang, Y.; Li, T.Z.; Kong, J. and Han, Z.H. (2008). Effects of leaf-applied potassium, gibberellin and source-sink ratio on potassium absorption and distribution in grape fruits. Scientia Hort., 115:164-167.

Pande, M.; Goli, M.B. and Bellaloui, N. (2014). Effect of foliar and soil application of potassium fertilizer on soybean seed protein, oil, fatty acids, and minerals. American Journal of Plant Sciences, 5: 541-548.

Peuke, A.D.; Jeschke, W.D. and Hartung, W. (2002). Flows of elements, ions and abscisic acid in Ricinus communis and site of nitrate reduction under potassium limitation. Journal of Experimental Botany, 53:241-250.

Pino, J.A.; Rosado, A. and Fuentes, V. (1996). Chemical composition of the seed oil of Coriandrum sativum L. from Cuba. J. Essent. Oil Res., 8: 97-98.

Rangappa, M.; Bhardwaj, H.L.; Showhda, M. and Hamama, A.A. (1997). Cilantro response to nitrogen fertilizer rates. J. Herbs Spices Med. Plants, 5:63-68.

Römheld, V. and Kirkby, E.A. (2010). Research on potassium in agriculture: Needs and prospects. Plant Soil, 335: 155-180.

Shin, R. (2014). Strategies for improving potassium use efficiency in plants. Mol. Cells, 37: 575-584.

Snedecor, G.W. and Cochran, W.G. (1989) Statistical Methods, $8^{\text {th }}$ ed. Iowa State University Press, Ames, 503 p.

Steel, R.G. and Torrie, T.H. (1982) Principles and Procedures of Statistics, $3^{\text {rd }}$ ed. McGraw-Hill International Book Co., London, 481 p.

Tiwari, R.S.; Agarwal, A. and Singar, S.C. (2002). Effect of dates of sowing and number of cuttings on growth, seed yield and economics of coriander cv. Pant Haritima. Crop Res. Hisar, 23:324-329.

Tripathi, M.L.; Trivedi, S.K. and Yadav, R.B. (2009). Effect of irrigation and nutrient levels on growth and yield of coriander (Coriandrum sativum L.). Indian J. Agron., 45 (4): 25-48.

Walker, D.J.; Leigh, R.A. and Miller, A.J. (1996). Potassium homeostasis in vacuolate plant cells. Proc. Natl. Acad. Sci. USA, 93: 10510-10514.

Wang, Y. and Wu, W.H. (2010). Plant sensing and signaling in response to $\mathrm{K}^{+}$deficiency. Mol. Plant., 3:280-287.

Weiss, E.A. (2002). Spice Crops. CABI Publishing, New York, USA, 432 p.

Zheljazkov, V.D.; Pickett, K.M.; Caldwell, C.D.; Pincock, J.A.; Roberts, J.C. and 


\section{E.Y. Abdul-Hafeez}

Mapplebeck, L. (2008). Cultivar and sowing date effects on seed field and oil composition of coriander in Atlantik Canada. Indust. Crops Prod., 28: 88-94.
Zörb, C.; Senbayram, M. and Peiter, E. (2014). Potassium in agriculture-status and perspectives. J. Plant Physiol., 171: 656-669.

المعاملة الخارجية بالبوتاسيوم داي هيدروجين أورثو فوسفات ومواعيد الزراعة تزيد من محصول الثمار والزيت الطيار للكزبرة فيثون

$$
\text { قسم الزينة، كلية الزرامة عصام، جامعة أسيوطف، أسيوط، مصر }
$$

أجريت تجربة حقلية على مدار موسمين لدراسة تأثير الرش الورقي بالبوتاسيوم داي هيدروجين أورثوفوسفات

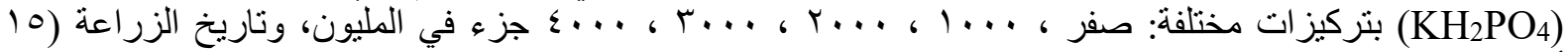

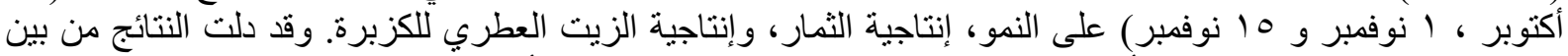

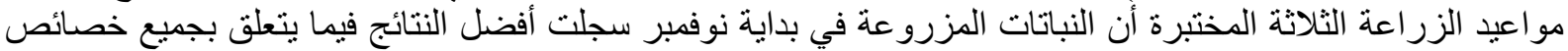

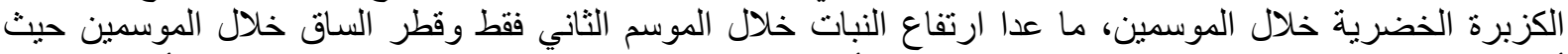

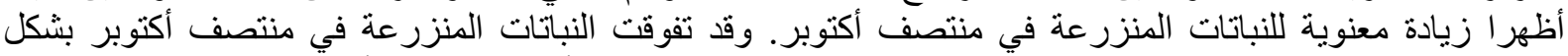

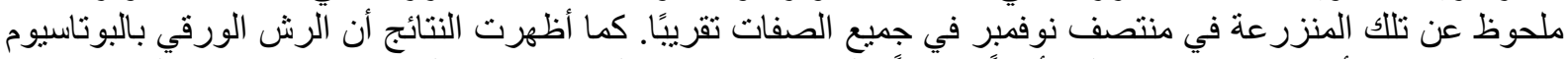

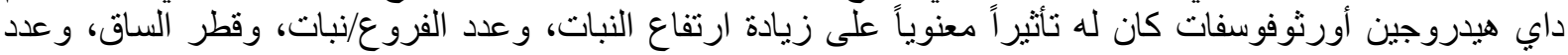

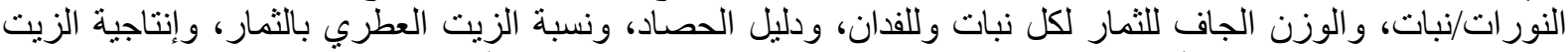

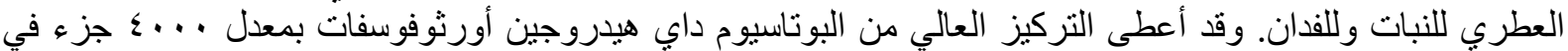

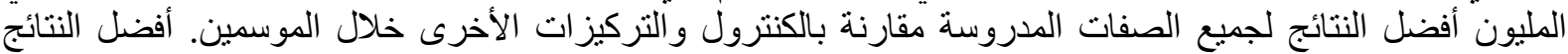

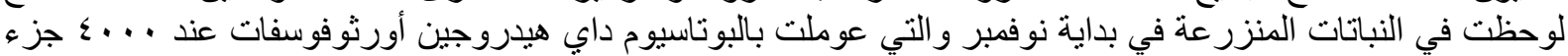

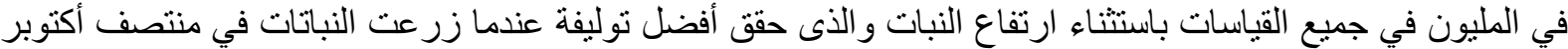

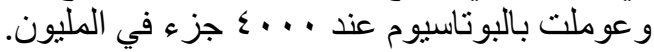

